SUPPLEMENTARY MATERIAL FOR

\title{
Switchable Solvents Consisting of Amidine/Alcohol or Guanidine/Alcohol Mixtures**
}

\author{
Lam Phan, ${ }^{\dagger}$ Daniel Chiu, ${ }^{\dagger}$ David J. Heldebrant, ${ }^{\dagger}$ Hillary Huttenhower, ${ }^{\ddagger}$ Ejae John, ${ }^{\ddagger}$ Xiaowang \\ Li, ${ }^{\dagger}$ Pamela Pollet, ${ }^{\dagger}$ Ruiyao Wang, Charles A. Eckert, ${ }^{\dagger}{ }^{\dagger}$ Charles L. Liotta, ${ }^{\ddagger}$ and Philip G. \\ Jessop* $*$ \\ Department of Chemistry, Queen's University, Kingston, Ontario, Canada and Schools of Chemistry \\ and Biochemistry and School of Chemical and Biomolecular Engineering, Georgia Institute of \\ Technology, Atlanta, Georgia 30332-0100 (USA) \\ * To whom correspondence should be addressed. Phone: (613) 533-3212. Fax (613) 533-6669. E- \\ mail: jessop@chem.queensu.ca \\ ${ }^{\dagger}$ Queen's University \\ * School of Chemistry and Biochemistry, Georgia Institute of Technology \\ ${ }^{\S}$ School of Chemical and Biomolecular Engineering, Georgia Institute of Techology
}

\section{Contents:}

Table S1. ${ }^{13} \mathrm{C}\left\{{ }^{1} \mathrm{H}\right\}$ NMR chemical shifts of $[\mathrm{DBUH}]\left[\mathrm{O}_{2} \mathrm{COR}\right]$ salts in $\mathrm{CDCl}_{3}$.

Table S2. ${ }^{13} \mathrm{C}\left\{{ }^{1} \mathrm{H}\right\}$ NMR chemical shifts of pure 1-alkanols in $\mathrm{CDCl}_{3}$.

Table S3. ${ }^{1} \mathrm{H}$ NMR chemical shifts of $[\mathrm{DBUH}]\left[\mathrm{O}_{2} \mathrm{COR}\right]$ salts in $\mathrm{CDCl}_{3}$.

Table S4. ${ }^{1} \mathrm{H}$ NMR chemical shifts of pure 1-alkanols in $\mathrm{CDCl}_{3}$.

Table S5. Melting points of neat base/ $\mathrm{ROH} / \mathrm{CO}_{2}$ salts.

Table S6. Polarities of switchable solvents as indicated by Nile Red solvatochromic dye.

Figure S1. Unit cell packing for $[\mathrm{DBUH}]\left[\mathrm{O}_{2} \mathrm{COCH}_{3}\right]$.

Table S7. Crystal data and structure refinement for $[\mathrm{DBUH}]\left[\mathrm{O}_{2} \mathrm{COCH}_{3}\right]$.

Table S8. Atomic coordinates and equivalent isotropic displacement parameters for $[\mathrm{DBUH}]\left[\mathrm{O}_{2} \mathrm{COCH}_{3}\right]$.

Table S9. Bond lengths and angles for $[\mathrm{DBUH}]\left[\mathrm{O}_{2} \mathrm{COCH}_{3}\right]$.

Table S10. Anisotropic displacement parameters for [DBUH] $\left[\mathrm{O}_{2} \mathrm{COCH}_{3}\right]$.

Table S11. Hydrogen coordinates and isotropic displacement parameters for $[\mathrm{DBUH}]\left[\mathrm{O}_{2} \mathrm{COCH}_{3}\right]$.

Table S12. Torsion angles for $[\mathrm{DBUH}]\left[\mathrm{O}_{2} \mathrm{COCH}_{3}\right]$.

Table S13. Hydrogen bonds for $[\mathrm{DBUH}]\left[\mathrm{O}_{2} \mathrm{COCH}_{3}\right]$. 
Table S1. ${ }^{13} \mathrm{C}\left\{{ }^{1} \mathrm{H}\right\}$ NMR chemical shifts of $[\mathrm{DBUH}]\left[\mathrm{O}_{2} \mathrm{COR}\right]$ salts in $\mathrm{CDCl}_{3}$.

\begin{tabular}{|c|c|c|c|c|c|c|c|}
\hline \multirow[t]{2}{*}{ Carbon } & & \multicolumn{6}{|l|}{$\mathrm{R}=$} \\
\hline & $\mathrm{H}$ & ethyl & propyl & 1-butyl & 1-hexyl & 1-octyl & 1-decyl \\
\hline carbonate & 161.1 & 158.6 & 158.6 & 157.8 & 158.7 & 157.5 & 158.3 \\
\hline $\mathrm{DBUC}_{2}$ & 53.2 & 53.5 & 53.9 & 52.9 & 53.5 & 52.6 & 53.3 \\
\hline $\mathrm{DBU} \mathrm{C}_{3}$ & 29.7 & 27.1 & 26.9 & 28.1 & 26.8 & 26.7 & 26.4 \\
\hline $\mathrm{DBUC}_{4}$ & 28.3 & 28.9 & 28.9 & 23.5 & 28.8 & 23.3 & 23.7 \\
\hline $\mathrm{DBUC}_{5}$ & 25.7 & 24.4 & 25.9 & 26.2 & 24.0 & 28.0 & 28.6 \\
\hline $\mathrm{DBU} \mathrm{C}_{6}$ & 36.6 & 33.1 & 31.5 & 31.0 & 32.3 & 31.8 & 31.7 \\
\hline $\mathrm{DBU} \mathrm{C}_{7}$ & 162.6 & 164.4 & 164.9 & 164.2 & 164.9 & 163.6 & 164.8 \\
\hline $\mathrm{DBU} \mathrm{C}_{9}$ & 43.1 & 39.4 & 38.4 & 38.1 & 38.6 & 38.2 & 38.0 \\
\hline $\mathrm{DBU}_{10}$ & 22.0 & 20.1 & 19.5 & 19.1 & 19.7 & 19.0 & 19.2 \\
\hline $\mathrm{DBU}_{11}$ & 48.5 & 48.2 & 48.5 & 47.5 & 48.1 & 47.2 & 47.8 \\
\hline $\mathrm{ROH} \mathrm{C}_{1}$ & N/A & 60.0 & 65.7 & 63.5 & 64.6 & 63.5 & 64.2 \\
\hline $\mathrm{ROH} \mathrm{C}_{2}$ & N/A & 15.1 & 31.5 & 31.7 & 29.4 & 28.6 & 28.6 \\
\hline $\mathrm{ROH} \mathrm{C}_{3}$ & N/A & N/A & 19.1 & 18.3 & 25.5 & 25.0 & 25.3 \\
\hline $\mathrm{ROH} \mathrm{C}_{4}$ & N/A & N/A & N/A & 13.0 & 31.5 & 28.0 & 29.0 \\
\hline $\mathrm{ROH} \mathrm{C}_{5}$ & N/A & N/A & N/A & N/A & 22.2 & 28.0 & 29.0 \\
\hline $\mathrm{ROH} \mathrm{C}_{6}$ & N/A & N/A & N/A & N/A & 13.7 & 30.5 & 29.0 \\
\hline $\mathrm{ROH} \mathrm{C}_{7}$ & N/A & N/A & N/A & N/A & N/A & 21.3 & 29.0 \\
\hline $\mathrm{ROH} \mathrm{C}_{8}$ & N/A & $\mathrm{N} / \mathrm{A}$ & N/A & N/A & N/A & 12.8 & 31.1 \\
\hline $\mathrm{ROH} \mathrm{C}_{9}$ & N/A & N/A & N/A & N/A & $\mathrm{N} / \mathrm{A}$ & $\mathrm{N} / \mathrm{A}$ & 21.9 \\
\hline $\mathrm{ROH} \mathrm{C}_{10}$ & N/A & N/A & N/A & N/A & N/A & N/A & 13.4 \\
\hline
\end{tabular}

Table S2. ${ }^{13} \mathrm{C}\left\{{ }^{1} \mathrm{H}\right\}$ NMR chemical shifts of pure 1-alkanols in $\mathrm{CDCl}_{3}$.

\begin{tabular}{lllllll}
\hline Carbon & Ethanol & Propanol & Butanol & Hexanol & Octanol & Decanol \\
\hline ROH C1 & 58.0 & 64.5 & 62.4 & 62.7 & 62.9 & 62.9 \\
ROH C2 & 18.3 & 26.0 & 34.8 & 32.7 & 32.9 & 32.8 \\
ROH C3 & na & 10.2 & 19.0 & 25.4 & 25.8 & 25.9 \\
ROH C4 & na & na & 13.9 & 31.6 & 29.3 & 29.6 \\
ROH C5 & na & na & na & 22.5 & 29.4 & 29.6 \\
ROH C6 & na & na & na & 14.0 & 31.8 & 29.6 \\
ROH C7 & na & na & na & na & 22.7 & 29.4 \\
ROH C8 & na & na & na & na & 14.1 & 32.0 \\
ROH C9 & na & na & na & na & na & 22.8 \\
ROH C10 & na & na & na & na & na & 14.2 \\
\hline
\end{tabular}


Table S3. ${ }^{1} \mathrm{H}$ NMR chemical shifts of $[\mathrm{DBUH}]\left[\mathrm{O}_{2} \mathrm{COR}\right]$ salts in $\mathrm{CDCl}_{3}$.

\begin{tabular}{|c|c|c|c|c|c|c|c|}
\hline \multirow[b]{2}{*}{ Carbon } & \multicolumn{7}{|l|}{$\mathrm{R}=$} \\
\hline & $\mathrm{H}$ & ethyl & 1-propyl & 1-butyl & 1-hexyl & 1-octyl & 1-decyl \\
\hline DBU C2 & 3.39 & 3.61 & 3.60 & 3.54 & 3.49 & 3.53 & 3.53 \\
\hline DBU C3 & 1.72 & 1.74 & 1.75 & 1.73 & 1.75 & 1.70 & 1.76 \\
\hline DBU C4 & 1.65 & 1.74 & 1.75 & 1.73 & 1.75 & 1.60 & 1.76 \\
\hline DBU C5 & 1.72 & 1.74 & 1.75 & 1.73 & 1.75 & 1.75 & 1.76 \\
\hline DBU C6 & 2.71 & 2.86 & 2.86 & 2.79 & 2.81 & 2.77 & 2.84 \\
\hline DBU C9 & 3.39 & 3.42 & 3.43 & 3.40 & 3.43 & 3.39 & 3.43 \\
\hline DBU C10 & 1.94 & 2.05 & 2.06 & 2.01 & 2.00 & 2.00 & 2.02 \\
\hline DBU C11 & 3.39 & 3.61 & 3.61 & 3.54 & 3.49 & 3.53 & 3.53 \\
\hline ROH C1 & na & 3.91 & 3.84 & 3.88 & 3.90 & 3.88 & 3.89 \\
\hline $\mathrm{ROH} \mathrm{C} 2$ & na & 1.19 & 1.60 & 1.60 & 1.58 & 1.58 & 1.59 \\
\hline $\mathrm{ROH} \mathrm{C} 3$ & na & na & 0.95 & 1.41 & 1.37 & 1.36 & 1.27 \\
\hline ROH C4 & na & na & na & 0.92 & 1.29 & 1.28 & 1.27 \\
\hline ROH C5 & na & na & na & na & 1.29 & 1.28 & 1.27 \\
\hline ROH C6 & na & na & na & na & 0.87 & 1.28 & 1.27 \\
\hline $\mathrm{ROH} \mathrm{C7}$ & na & na & na & na & na & 1.28 & 1.27 \\
\hline $\mathrm{ROH} \mathrm{C8}$ & na & na & na & na & na & 0.88 & 1.27 \\
\hline ROH C9 & na & na & na & na & na & na & 1.27 \\
\hline ROH C10 & na & na & na & na & na & na & 0.88 \\
\hline
\end{tabular}

na $=$ not applicable. Strongly overlapped peaks have been given identical chemical shifts.

Table S4. ${ }^{1} \mathrm{H}$ NMR chemical shifts of pure 1-alkanols in $\mathrm{CDCl}_{3}$.

\begin{tabular}{lllllll}
\hline Proton & EtOH & PrOH & BuOH & Hexanol & Octanol & Decanol \\
\hline ROH C1 & 3.68 & 3.58 & 3.62 & 3.63 & 3.62 & 3.61 \\
ROH C2 & 1.22 & 1.58 & 1.55 & 1.58 & 1.56 & 1.56 \\
ROH C3 & na & 0.93 & 1.38 & 1.30 & 1.30 & 1.27 \\
ROH C4 & na & na & 0.93 & 1.30 & 1.30 & 1.27 \\
ROH C5 & na & na & na & 1.30 & 1.30 & 1.27 \\
ROH C6 & na & na & na & 0.91 & 1.30 & 1.27 \\
ROH C7 & na & na & na & na & 1.30 & 1.27 \\
ROH C8 & na & na & na & na & 0.88 & 1.27 \\
ROH C9 & na & na & na & na & na & 1.31 \\
ROH C10 & na & na & na & na & na & 0.88 \\
\hline
\end{tabular}


Table S5. Melting points of neat base $/ \mathrm{ROH} / \mathrm{CO}_{2}$ salts. $^{a}$

\begin{tabular}{lll}
\hline n-Alcohol & $\mathrm{T}_{\mathrm{m}}$ (DBU salt ${ }^{\circ} \mathrm{C}$ & $\mathrm{T}_{\mathrm{m}}(\mathrm{TMBG}$ salt $){ }^{\circ} \mathrm{C}$ \\
\hline Water & $115^{b}$ & $\mathrm{nd}$ \\
Methanol & 59 & -24.5 \\
Ethanol & 34 & $\mathrm{nd}$ \\
Propanol & 5 & $\mathrm{nd}$ \\
Butanol & 6 & -23 \\
Hexanol & 22 & $\mathrm{nd}$ \\
Octanol & 30 & -17.5 \\
Decanol & 37 & $\mathrm{nd}$ \\
Dodecanol & nd & -10.5 \\
\hline
\end{tabular}

${ }^{a}$ This work except as noted. "nd" = not determined.

${ }^{b}$ Reference: Hori, Y.; Nagano, Y.; Nakau, J.; Taniguchi, H., Chemistry Express (Kinki Chemical Society) (1986) 1, 173-176.

Table S6. Polarities of switchable solvents as indicated by Nile Red solvatochromic dye.

\begin{tabular}{llll}
\hline Base & n-Alcohol & $\begin{array}{l}\lambda_{\max } \text { (neutral solvent) } \\
\mathrm{nm}\end{array}$ & $\begin{array}{l}\lambda_{\max } \text { (ionic solvent) } \\
\mathrm{nm}\end{array}$ \\
\hline TMBG & Dodecanol & 528.0 & 536.5 \\
TMBG & Octanol & 530.1 & 541.1 \\
TMBG & Hexanol & 531.1 & 545.0 \\
TMBG & Butanol & 532.0 & 544.4 \\
TMBG & Methanol & 538.0 & 554.0 \\
DBU & Decanol & 537 & 540 \\
DBU & Octanol & 537 & 542 \\
DBU & Hexanol & 537 & 544 \\
DBU & Butanol & 538 & 548 \\
DBU & Propanol & 542 & 552 \\
\hline
\end{tabular}




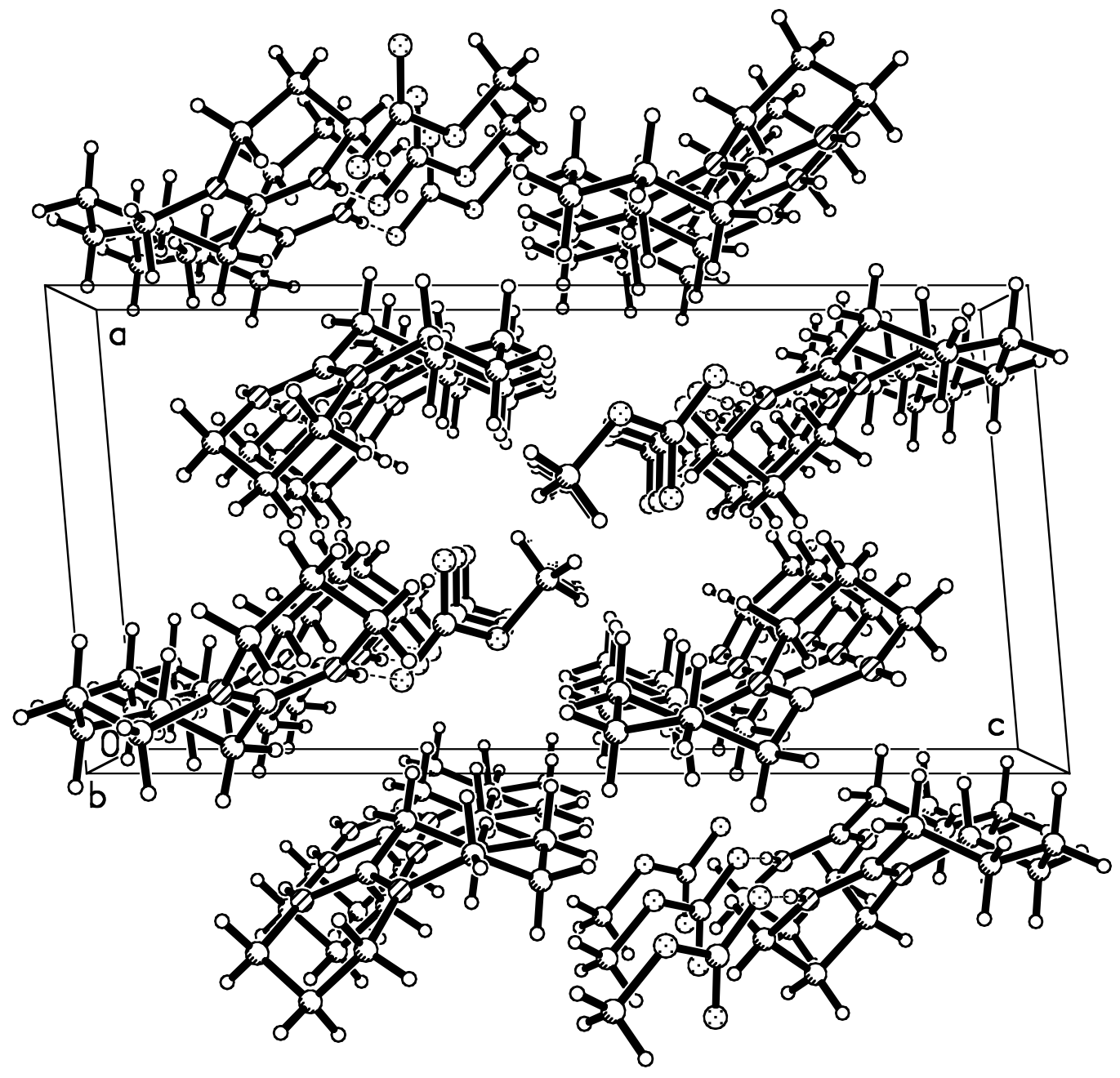

Figure S1. Unit cell packing for $[\mathrm{DBUH}]\left[\mathrm{O}_{2} \mathrm{COCH}_{3}\right]$. 
Table S7. Crystal data and structure refinement for $[\mathrm{DBUH}]\left[\mathrm{O}_{2} \mathrm{COCH}_{3}\right]$.

Empirical formula

Formula weight

Temperature

Wavelength

Crystal system

Space group

Unit cell dimensions

Volume

Z

Density (calculated)

Absorption coefficient

$\mathrm{F}(000)$

Crystal size

Theta range for data collection

Index ranges

Reflections collected

Independent reflections

Completeness to theta $=25.00^{\circ}$

Absorption correction

Max. and min. transmission

Refinement method

Data / restraints / parameters

Goodness-of-fit on $\mathrm{F}^{2}$

Final $\mathrm{R}$ indices $[\mathrm{I}>2 \operatorname{sigma}(\mathrm{I})]$

$\mathrm{R}$ indices (all data)

Largest diff. peak and hole
C11 H20 N2 O3

228.29

180(2) K

$0.71073 \AA$

Monoclinic

P2(1)/c

$\mathrm{a}=8.9898(10) \AA$

$\alpha=90^{\circ}$.

$\mathrm{b}=7.4386(8) \AA$

$\beta=94.892(2)^{\circ}$.

$\mathrm{c}=18.080(2) \AA$

$\gamma=90^{\circ}$.
4

$1.259 \mathrm{Mg} / \mathrm{m}^{3}$

$0.092 \mathrm{~mm}^{-1}$

496

$0.35 \times 0.25 \times 0.08 \mathrm{~mm}^{3}$

2.26 to $25.00^{\circ}$.

$-10<=\mathrm{h}<=10,-8<=\mathrm{k}<=8,-20<=\mathrm{l}<=21$

6837

$2120[\mathrm{R}(\mathrm{int})=0.0233]$

$99.9 \%$

Empirical (Bruker SADABS)

1.0000 and 0.8311

Full-matrix least-squares on $\mathrm{F}^{2}$

$2120 / 0 / 225$

1.000

$\mathrm{R} 1=0.0303, \mathrm{wR} 2=0.0592$

$\mathrm{R} 1=0.0462, \mathrm{wR} 2=0.0632$

0.122 and -0.151 e. $\AA^{-3}$ 
Table S8. Atomic coordinates (x 10 $)$ and equivalent isotropic displacement parameters $\left(\AA^{2} \times 10^{3}\right)$ for $[\mathrm{DBUH}]\left[\mathrm{O}_{2} \mathrm{COCH}_{3}\right]$. U(eq) is defined as one third of the trace of the orthogonalized Uij tensor.

\begin{tabular}{lcccc}
\hline & $\mathrm{x}$ & $\mathrm{y}$ & $\mathrm{z}$ & $\mathrm{U}(\mathrm{eq})$ \\
\hline $\mathrm{N}(1)$ & $-2026(1)$ & $4539(1)$ & $3185(1)$ & $33(1)$ \\
$\mathrm{N}(2)$ & $-2376(1)$ & $2672(1)$ & $2169(1)$ & $36(1)$ \\
$\mathrm{C}(1)$ & $-1796(1)$ & $3000(2)$ & $2847(1)$ & $31(1)$ \\
$\mathrm{C}(2)$ & $-868(2)$ & $1559(2)$ & $3233(1)$ & $35(1)$ \\
$\mathrm{C}(3)$ & $-1528(2)$ & $809(2)$ & $3922(1)$ & $41(1)$ \\
$\mathrm{C}(4)$ & $-1232(2)$ & $1960(2)$ & $4614(1)$ & $44(1)$ \\
$\mathrm{C}(5)$ & $-1882(2)$ & $3846(2)$ & $4546(1)$ & $41(1)$ \\
$\mathrm{C}(6)$ & $-1294(2)$ & $4942(2)$ & $3928(1)$ & $38(1)$ \\
$\mathrm{C}(7)$ & $-3045(2)$ & $5920(2)$ & $2841(1)$ & $38(1)$ \\
$\mathrm{C}(8)$ & $-4120(2)$ & $5124(2)$ & $2247(1)$ & $41(1)$ \\
$\mathrm{C}(9)$ & $-3300(2)$ & $3965(2)$ & $1730(1)$ & $42(1)$ \\
$\mathrm{O}(1)$ & $-2826(1)$ & $-2364(1)$ & $683(1)$ & $36(1)$ \\
$\mathrm{O}(2)$ & $-4393(1)$ & $-340(1)$ & $1116(1)$ & $47(1)$ \\
$\mathrm{O}(3)$ & $-2061(1)$ & $-674(1)$ & $1630(1)$ & $41(1)$ \\
$\mathrm{C}(10)$ & $-3152(2)$ & $-1020(1)$ & $1173(1)$ & $30(1)$ \\
$\mathrm{C}(11)$ & $-4012(2)$ & $-2814(2)$ & $139(1)$ & $46(1)$ \\
& & & & \\
\hline
\end{tabular}


Table S9. Bond lengths $[\AA]$ and angles $\left[^{\circ}\right]$ for $[\mathrm{DBUH}]\left[\mathrm{O}_{2} \mathrm{COCH}_{3}\right]$.

\begin{tabular}{|c|c|}
\hline $\mathrm{N}(1)-\mathrm{C}(1)$ & $1.3224(14)$ \\
\hline $\mathrm{N}(1)-\mathrm{C}(6)$ & $1.4759(17)$ \\
\hline $\mathrm{N}(1)-\mathrm{C}(7)$ & $1.4776(16)$ \\
\hline $\mathrm{N}(2)-\mathrm{C}(1)$ & $1.3128(15)$ \\
\hline $\mathrm{N}(2)-\mathrm{C}(9)$ & $1.4610(16)$ \\
\hline $\mathrm{C}(1)-\mathrm{C}(2)$ & $1.4946(16)$ \\
\hline $\mathrm{C}(2)-\mathrm{C}(3)$ & $1.5289(19)$ \\
\hline$C(3)-C(4)$ & $1.5214(19)$ \\
\hline$C(4)-C(5)$ & $1.521(2)$ \\
\hline$C(5)-C(6)$ & $1.5146(19)$ \\
\hline$C(7)-C(8)$ & $1.5035(19)$ \\
\hline $\mathrm{C}(8)-\mathrm{C}(9)$ & $1.509(2)$ \\
\hline $\mathrm{O}(1)-\mathrm{C}(10)$ & $1.3838(14)$ \\
\hline $\mathrm{O}(1)-\mathrm{C}(11)$ & $1.4283(16)$ \\
\hline $\mathrm{O}(2)-\mathrm{C}(10)$ & $1.2211(14)$ \\
\hline $\mathrm{O}(3)-\mathrm{C}(10)$ & $1.2539(14)$ \\
\hline $\mathrm{C}(1)-\mathrm{N}(1)-\mathrm{C}(6)$ & $121.40(10)$ \\
\hline $\mathrm{C}(1)-\mathrm{N}(1)-\mathrm{C}(7)$ & $121.58(11)$ \\
\hline $\mathrm{C}(6)-\mathrm{N}(1)-\mathrm{C}(7)$ & $117.01(10)$ \\
\hline $\mathrm{C}(1)-\mathrm{N}(2)-\mathrm{C}(9)$ & $123.18(11)$ \\
\hline $\mathrm{N}(2)-\mathrm{C}(1)-\mathrm{N}(1)$ & $121.76(11)$ \\
\hline $\mathrm{N}(2)-\mathrm{C}(1)-\mathrm{C}(2)$ & $117.69(11)$ \\
\hline $\mathrm{N}(1)-\mathrm{C}(1)-\mathrm{C}(2)$ & $120.55(11)$ \\
\hline $\mathrm{C}(1)-\mathrm{C}(2)-\mathrm{C}(3)$ & $113.71(11)$ \\
\hline$C(4)-C(3)-C(2)$ & $114.33(12)$ \\
\hline$C(5)-C(4)-C(3)$ & $114.39(12)$ \\
\hline$C(6)-C(5)-C(4)$ & $113.61(12)$ \\
\hline $\mathrm{N}(1)-\mathrm{C}(6)-\mathrm{C}(5)$ & $113.96(11)$ \\
\hline $\mathrm{N}(1)-\mathrm{C}(7)-\mathrm{C}(8)$ & $111.37(10)$ \\
\hline $\mathrm{C}(7)-\mathrm{C}(8)-\mathrm{C}(9)$ & $110.44(13)$ \\
\hline $\mathrm{N}(2)-\mathrm{C}(9)-\mathrm{C}(8)$ & $108.96(11)$ \\
\hline $\mathrm{C}(10)-\mathrm{O}(1)-\mathrm{C}(11)$ & $115.07(10)$ \\
\hline $\mathrm{O}(2)-\mathrm{C}(10)-\mathrm{O}(3)$ & $128.93(12)$ \\
\hline $\mathrm{O}(2)-\mathrm{C}(10)-\mathrm{O}(1)$ & $119.15(11)$ \\
\hline $\mathrm{O}(3)-\mathrm{C}(10)-\mathrm{O}(1)$ & $111.92(11)$ \\
\hline
\end{tabular}

Symmetry transformations used to generate equivalent atoms: 
Table S10. Anisotropic displacement parameters $\left(\AA^{2} \times 10^{3}\right)$ for $[\mathrm{DBUH}]\left[\mathrm{O}_{2} \mathrm{COCH}_{3}\right]$. The anisotropic displacement factor exponent takes the form: $-2 \pi^{2}\left[\mathrm{~h}^{2} \mathrm{a}^{* 2} \mathrm{U}^{11}+\ldots+2 \mathrm{~h} \mathrm{k} \mathrm{a}^{*} \mathrm{~b}^{*} \mathrm{U}^{12}\right]$

\begin{tabular}{lcccccc}
\hline & $\mathrm{U}^{11}$ & $\mathrm{U}^{22}$ & $\mathrm{U}^{33}$ & $\mathrm{U}^{23}$ & $\mathrm{U}^{13}$ & $\mathrm{U}^{12}$ \\
\hline $\mathrm{N}(1)$ & $35(1)$ & $27(1)$ & $36(1)$ & $-2(1)$ & $0(1)$ & $0(1)$ \\
$\mathrm{N}(2)$ & $46(1)$ & $31(1)$ & $32(1)$ & $-1(1)$ & $0(1)$ & $1(1)$ \\
$\mathrm{C}(1)$ & $29(1)$ & $29(1)$ & $35(1)$ & $0(1)$ & $6(1)$ & $-5(1)$ \\
$\mathrm{C}(2)$ & $34(1)$ & $31(1)$ & $40(1)$ & $-5(1)$ & $-1(1)$ & $2(1)$ \\
$\mathrm{C}(3)$ & $44(1)$ & $35(1)$ & $44(1)$ & $5(1)$ & $-6(1)$ & $-1(1)$ \\
$\mathrm{C}(4)$ & $40(1)$ & $53(1)$ & $37(1)$ & $4(1)$ & $-3(1)$ & $-1(1)$ \\
$\mathrm{C}(5)$ & $33(1)$ & $55(1)$ & $35(1)$ & $-7(1)$ & $0(1)$ & $2(1)$ \\
$\mathrm{C}(6)$ & $35(1)$ & $34(1)$ & $44(1)$ & $-10(1)$ & $-1(1)$ & $-1(1)$ \\
$\mathrm{C}(7)$ & $41(1)$ & $29(1)$ & $44(1)$ & $5(1)$ & $7(1)$ & $2(1)$ \\
$\mathrm{C}(8)$ & $42(1)$ & $37(1)$ & $42(1)$ & $10(1)$ & $1(1)$ & $2(1)$ \\
$\mathrm{C}(9)$ & $51(1)$ & $39(1)$ & $35(1)$ & $6(1)$ & $-2(1)$ & $1(1)$ \\
$\mathrm{O}(1)$ & $38(1)$ & $30(1)$ & $41(1)$ & $-6(1)$ & $3(1)$ & $4(1)$ \\
$\mathrm{O}(2)$ & $34(1)$ & $57(1)$ & $50(1)$ & $-15(1)$ & $0(1)$ & $12(1)$ \\
$\mathrm{O}(3)$ & $36(1)$ & $41(1)$ & $45(1)$ & $-7(1)$ & $-5(1)$ & $4(1)$ \\
$\mathrm{C}(10)$ & $33(1)$ & $27(1)$ & $32(1)$ & $2(1)$ & $6(1)$ & $1(1)$ \\
$\mathrm{C}(11)$ & $49(1)$ & $40(1)$ & $46(1)$ & $-12(1)$ & $-1(1)$ & $0(1)$ \\
& & & & & & \\
\hline
\end{tabular}


Table S11. Hydrogen coordinates (x $\left.10^{4}\right)$ and isotropic displacement parameters $\left(\AA^{2} \times 10^{3}\right)$ for $[\mathrm{DBUH}]\left[\mathrm{O}_{2} \mathrm{COCH}_{3}\right]$.

\begin{tabular}{|c|c|c|c|c|}
\hline & $\mathrm{x}$ & $\mathrm{y}$ & z & $\mathrm{U}(\mathrm{eq})$ \\
\hline $\mathrm{H}(2 \mathrm{~N})$ & $-2283(15)$ & $1540(20)$ & 1982(7) & $51(4)$ \\
\hline $\mathrm{H}(2 \mathrm{~A})$ & $-769(13)$ & $610(15)$ & 2881(7) & $35(3)$ \\
\hline $\mathrm{H}(2 \mathrm{~B})$ & 139(14) & $2020(15)$ & $3362(6)$ & $37(3)$ \\
\hline $\mathrm{H}(3 \mathrm{~A})$ & $-1088(15)$ & $-362(17)$ & 4017(7) & $43(4)$ \\
\hline $\mathrm{H}(3 \mathrm{~B})$ & $-2645(16)$ & $609(15)$ & $3810(7)$ & $42(4)$ \\
\hline $\mathrm{H}(4 \mathrm{~A})$ & $-1653(15)$ & $1346(18)$ & $5056(8)$ & $55(4)$ \\
\hline $\mathrm{H}(4 \mathrm{~B})$ & $-140(16)$ & $2088(16)$ & 4721(7) & $43(4)$ \\
\hline $\mathrm{H}(5 \mathrm{~A})$ & $-2971(15)$ & $3796(16)$ & 4474(6) & $40(4)$ \\
\hline $\mathrm{H}(5 \mathrm{~B})$ & $-1599(14)$ & $4477(16)$ & $5020(8)$ & $48(4)$ \\
\hline $\mathrm{H}(6 \mathrm{~A})$ & $-183(15)$ & 4791(15) & $3904(6)$ & $38(4)$ \\
\hline $\mathrm{H}(6 \mathrm{~B})$ & $-1485(13)$ & $6253(17)$ & 3992(6) & $41(3)$ \\
\hline $\mathrm{H}(7 \mathrm{~A})$ & $-2420(13)$ & $6861(17)$ & $2634(6)$ & $38(3)$ \\
\hline $\mathrm{H}(7 \mathrm{~B})$ & $-3576(14)$ & $6419(16)$ & $3243(7)$ & $39(4)$ \\
\hline $\mathrm{H}(8 \mathrm{~A})$ & $-4850(16)$ & 4404(17) & 2471(8) & $47(4)$ \\
\hline $\mathrm{H}(8 \mathrm{~B})$ & $-4622(14)$ & $6093(17)$ & 1960(7) & $47(4)$ \\
\hline $\mathrm{H}(9 \mathrm{~A})$ & $-2652(15)$ & 4731(17) & 1439(7) & $48(4)$ \\
\hline $\mathrm{H}(9 \mathrm{~B})$ & $-3976(14)$ & $3293(17)$ & $1382(7)$ & $42(4)$ \\
\hline $\mathrm{H}(11 \mathrm{~A})$ & $-4848(19)$ & $-3350(20)$ & $408(8)$ & $73(5)$ \\
\hline $\mathrm{H}(11 \mathrm{~B})$ & $-4368(15)$ & $-1771(19)$ & $-142(7)$ & $50(4)$ \\
\hline $\mathrm{H}(11 \mathrm{C})$ & $-3597(17)$ & $-3740(20)$ & $-180(8)$ & $66(4)$ \\
\hline
\end{tabular}


Table S12. Torsion angles $\left[^{\circ}\right]$ for $[\mathrm{DBUH}]\left[\mathrm{O}_{2} \mathrm{COCH}_{3}\right]$.

\begin{tabular}{lc}
\hline $\mathrm{C}(9)-\mathrm{N}(2)-\mathrm{C}(1)-\mathrm{N}(1)$ & $0.76(19)$ \\
$\mathrm{C}(9)-\mathrm{N}(2)-\mathrm{C}(1)-\mathrm{C}(2)$ & $-179.85(12)$ \\
$\mathrm{C}(6)-\mathrm{N}(1)-\mathrm{C}(1)-\mathrm{N}(2)$ & $-176.11(11)$ \\
$\mathrm{C}(7)-\mathrm{N}(1)-\mathrm{C}(1)-\mathrm{N}(2)$ & $4.84(18)$ \\
$\mathrm{C}(6)-\mathrm{N}(1)-\mathrm{C}(1)-\mathrm{C}(2)$ & $4.52(18)$ \\
$\mathrm{C}(7)-\mathrm{N}(1)-\mathrm{C}(1)-\mathrm{C}(2)$ & $-174.53(12)$ \\
$\mathrm{N}(2)-\mathrm{C}(1)-\mathrm{C}(2)-\mathrm{C}(3)$ & $-115.88(13)$ \\
$\mathrm{N}(1)-\mathrm{C}(1)-\mathrm{C}(2)-\mathrm{C}(3)$ & $63.52(16)$ \\
$\mathrm{C}(1)-\mathrm{C}(2)-\mathrm{C}(3)-\mathrm{C}(4)$ & $-80.88(16)$ \\
$\mathrm{C}(2)-\mathrm{C}(3)-\mathrm{C}(4)-\mathrm{C}(5)$ & $61.24(18)$ \\
$\mathrm{C}(3)-\mathrm{C}(4)-\mathrm{C}(5)-\mathrm{C}(6)$ & $-59.57(18)$ \\
$\mathrm{C}(1)-\mathrm{N}(1)-\mathrm{C}(6)-\mathrm{C}(5)$ & $-70.66(16)$ \\
$\mathrm{C}(7)-\mathrm{N}(1)-\mathrm{C}(6)-\mathrm{C}(5)$ & $108.43(13)$ \\
$\mathrm{C}(4)-\mathrm{C}(5)-\mathrm{C}(6)-\mathrm{N}(1)$ & $80.53(16)$ \\
$\mathrm{C}(1)-\mathrm{N}(1)-\mathrm{C}(7)-\mathrm{C}(8)$ & $20.09(18)$ \\
$\mathrm{C}(6)-\mathrm{N}(1)-\mathrm{C}(7)-\mathrm{C}(8)$ & $-159.01(12)$ \\
$\mathrm{N}(1)-\mathrm{C}(7)-\mathrm{C}(8)-\mathrm{C}(9)$ & $-48.17(16)$ \\
$\mathrm{C}(1)-\mathrm{N}(2)-\mathrm{C}(9)-\mathrm{C}(8)$ & $-30.00(18)$ \\
$\mathrm{C}(7)-\mathrm{C}(8)-\mathrm{C}(9)-\mathrm{N}(2)$ & $52.16(16)$ \\
$\mathrm{C}(11)-\mathrm{O}(1)-\mathrm{C}(10)-\mathrm{O}(2)$ & $0.63(16)$ \\
$\mathrm{C}(11)-\mathrm{O}(1)-\mathrm{C}(10)-\mathrm{O}(3)$ & $-179.14(12)$ \\
\hline & \\
\hline
\end{tabular}

Symmetry transformations used to generate equivalent atoms: 
Table S13. Hydrogen bonds for $[\mathrm{DBUH}]\left[\mathrm{O}_{2} \mathrm{COCH}_{3}\right]\left[\AA\right.$ and $\left.^{\circ}\right]$.

\begin{tabular}{lcccc}
\hline D-H...A & d(D-H) & d(H...A & $d(D \ldots A)$ & $<($ DHA $)$ \\
\hline $\mathrm{N}(2)-\mathrm{H}(2 \mathrm{~N}) \ldots \mathrm{O}(3)$ & $0.911(14)$ & $1.785(15)$ & $2.6964(14)$ & $178.6(14)$ \\
\hline
\end{tabular}

Symmetry transformations used to generate equivalent atoms: 